\title{
Multiplicative Fault Estimation-Based Adaptive Sliding Mode Fault-Tolerant Control Design for Nonlinear Systems
}

\author{
Ali Ben Brahim (D), Slim Dhahri, Fayçal Ben Hmida, and Anis Sellami \\ Engineering Laboratory of Industrial Systems and Renewable Energies, National Higher School of Engineers of Tunis, Tunis \\ University, Tunis, Tunisia \\ Correspondence should be addressed to Ali Ben Brahim; alibenibrahimm@yahoo.com
}

Received 12 January 2018; Accepted 24 April 2018; Published 26 June 2018

Academic Editor: Chen Lu

Copyright ( 2018 Ali Ben Brahim et al. This is an open access article distributed under the Creative Commons Attribution License, which permits unrestricted use, distribution, and reproduction in any medium, provided the original work is properly cited.

\begin{abstract}
This article deals with the sliding mode fault-tolerant control (FTC) problem for a nonlinear system described under TakagiSugeno (T-S) fuzzy representation. In particular, the nonlinear system is corrupted with multiplicative actuator faults, process faults, and uncertainties. We start by constructing the separated FTC design to ensure robust stability of the closed-loop nonlinear system. First, we propose to conceive an adaptive observer in order to estimate nonlinear system states, as well as robust multiplicative fault estimation. The novelty of the proposed approach is that the observer gains are obtained by solving the multiobjective linear matrix inequality (LMI) optimization problem. Second, an adaptive sliding mode controller is suggested to offer a solution to stabilize the closed-loop system despite the occurrence of real fault effects. Compared with the separated FTC, this paper provides an integrated sliding mode FTC in order to achieve an optimal robustness interaction between observer and controller models. Thus, in a single-step LMI formulation, sufficient conditions are developed with multiobjective optimization performances to guarantee the stability of the closed-loop system. At last, nonlinear simulation results are given to illustrate the effectiveness of the proposed FTC to treat multiplicative faults.
\end{abstract}

\section{Introduction}

In the last few years, there has been a growing interest in fault-tolerant control (FTC) design based on a fault estimation (FE) technique (location, occurrence time, and magnitude). Active FTC takes a primordial place in modern control application, system reliability, and supervision of industrial technology.

The main challenge of active FTC is to conceive a robust controller such that the closed-loop system is stable with acceptable performances even with the presence simultaneously of faults and uncertainties. In the literature, several approaches have been proposed to explore this powerful issue (see for instance [1-6] and the references herein). In practical applications, most of the systems are complex and usually having hard nonlinearities, so it is significant to study FE and FTC for nonlinear systems. Since their excellent ability to describe a nonlinear system, very interesting approaches have represented nonlinear systems under the T-S fuzzy form [7]. Actually, in the presence of system uncertainties, several attempts have been oriented to the fault diagnosis and FTC of nonlinear systems (see for instance [8$13]$ and the references herein.

Popular FE approaches have been developed in a precise and effective way for nonlinear systems, where fault is modelled as additive changes appearing in actuators or sensors [14-18]. The major drawback of the preceding approaches resides primarily on treating actuator and sensor faults with additive terms. However, in practical engineering, it is often the case when some actuator faults and component faults occur in a multiplicative form. Thus, multiplicative faults are mixed with the inputs and outputs of the system. In this way, estimation of the characteristic and magnitude of unknown multiplicative faults has been a growing interest in modern control theory in recent years. It is practically important to decouple their eventual parameter or structure effects in the system or in the process model subject to ameliorate FTC design for a large class of nonlinear systems. Special attentions have already been made in the application of observer design to achieve multiplicative FE for linear and 
nonlinear systems $[19,20]$. In [21-24], robust observers have been used to estimate unknown fault for linear systems. The developed schemes are based on treating multiplicative faults with additive terms. Nevertheless, this approach may not be practical in all situations. For this purpose, it is not an easy task; real effect multiplicative fault detection, location, and estimation especially for nonlinear systems have been a significant research activity in the past decade. Zhang et al. [25] considers the problem of fault diagnosis for a class of nonlinear systems with unstructured modeling uncertainty. The proposed approach addresses the detection and isolation of nonlinear fault function that are modeled as measurable signals. More recently, in [26], a robust adaptive observer $\mathrm{FE}$ approach is discussed in order to extract the real component fault effects for Lipschitz nonlinear systems subject to unknown disturbances.

1.1. Contributions. Regarding the fact that multiplicative faults have not yet been fully tackled, an FE-based adaptive sliding mode FTC scheme for a class of uncertain nonlinear systems, approximated by the T-S representation, is of great interest in this paper. Thus, sliding mode control has been widely studied and employed in industrial applications based on its computational simplicity and in particular strong robustness against uncertainties or disturbances. The main contributions of the present paper are divided as follows:

(1) In the first scheme, we propose a separated sliding mode FTC for the closed-loop nonlinear system subject to both multiplicative faults and uncertainties. More precisely, it should be pointing that we consider multiplicative faults a partial loss of actuator effectiveness and parameter changes in the nonlinear system state matrix.

(2) In the second scheme, this paper provides an integrated sliding mode FTC in order to achieve an optimal robustness interaction between observer and controller models. Thus, in a single-step LMI formulation, sufficient conditions are developed with $H_{\infty}$ optimization performances to guarantee the stability of the closed-loop system. In particular, the fault nonlinear function satisfies a Lipschitz condition. In this study, we use a multiobjective LMI optimization approach in which the Lipschitz constant and uncertainty attenuation level are maximized simultaneously.

The remainder of this paper is organized as follows: Section 2 gives the description of the nonlinear system. In Section 3, we describe the proposed T-S adaptive observer design. Section 4 presents the sliding mode controller structure. Sections 5 and 6 propose, respectively, the design of separated and integrated sliding mode FTC schemes to stabilize the closed-loop system. The simulation example is given in Section 7 based on nonlinear simulation illustrating the effectiveness of the proposed schemes. Finally, Section 8 presents some concluding remarks.

\section{Problem Formulation}

Consider an uncertain nonlinear system governed by the following equations:

$$
\begin{aligned}
& \dot{x}(t)=\varphi_{1}(x(t), u(t), \xi(x, t), f(x, u, t)), \\
& y(t)=\varphi_{2}(x(t)),
\end{aligned}
$$

where $x(t) \in \mathrm{R}^{n}$ is the state vector, $u(t) \in \mathrm{R}^{m}$ represents the control inputs, $y(t) \in \mathrm{R}^{p}$ denotes the measurement output vector, and $\xi(x, t) \in \mathrm{R}^{l}$ stands for the uncertainty vector. In the present paper, $f(x, u, t) \in \mathrm{R}^{q}$ represents the component and/or actuator gain fault which is described as

$$
f(x, u, t)=\sum_{j=1}^{q} \boldsymbol{\theta}_{j}(t) h_{j}(x, u, t),
$$

where $\boldsymbol{\theta}_{j}(t)$ is a vector of unknown function reflecting the magnitude of the time-varying or constant multiplicative faults. $h_{j}(x, u, t)$ represents the functional structure of the $j t$ $\mathrm{h}$ multiplicative faults and usually mixes with system states and/or inputs. Before starting the main results of this paper, we will make the following assumptions.

Assumption 1. The fault vector $\boldsymbol{\theta}_{j}(t)$ is assumed to be unknown but bounded as $\left\|\theta_{j}(t)\right\| \leq\left\|\theta_{\max }\right\|=\rho, \forall j=1, \ldots, q$

, where $\theta_{\max } \in \mathrm{R}^{q}$ is a known constant vector and $\rho$ is a known positive constant.

Assumption 2. It is assumed that the nonlinear system states and inputs are all bounded before and after the occurrence of a fault, and fault nonlinear function structure $h_{j}(x, u, t)$ satisfies a Lipschitz condition locally on a set $M \subset \mathrm{R}^{n}$ in which

$$
\left\|h_{j}\left(x_{1}, u, t\right)-h_{j}\left(x_{2}, u, t\right)\right\| \leq \gamma_{j}\left\|x_{1}-x_{2}\right\|, \quad \forall x_{1}, x_{2} \in M,
$$

where $\gamma_{j}>0$ is called a Lipschitz constant and $j=1, \ldots, q .2 .1$. Design Objective. This paper features a robust estimation of real effect factor $\theta_{j}(t), \forall j=1, \ldots, q$, for the uncertain nonlinear system (1) subject both to multiplicative fault $f(x, u, t)$ given by (2) and to uncertainties $\xi(x, t)$. It was the main purpose of the paper to solve two problems by (i) estimating multiplicative fault magnitude using a robust adaptive observer and (ii) stabilizing the closed-loop nonlinear system, after the occurrence of multiplicative fault $f(x, u, t)$, using a robust adaptive sliding mode controller. To treat this powerful issue, this article introduces two different approaches: separated and integrated FE-based adaptive sliding mode FTC design.

We will make the following definition, notation, and lemma in obtaining the main results.

Definition. For an arbitrary matrix $X \in \mathrm{R}^{n \times m}$, if $X^{+} \in \mathrm{R}^{m \times n}$ verifies $X^{+} X=I_{m}$, then $X^{+}=\left(X^{T} X\right)^{-1} X^{T}$ is said to be the left_inverse of $X$. 
Notation. The notation $(*)$ corresponds to the symmetry matrix block, $\mathrm{He}(X)$ signifies $X+X^{T}$, and $\|$.$\| stands for$ the standard norm symbol.

Lemma 1. For matrices $\mathbf{X}$ and $\mathbf{Y}$ with appropriate dimensions, the following condition holds:

$$
X^{T} Y+Y^{T} X \leq \varepsilon^{-1} X^{T} X+\varepsilon Y^{T} Y,
$$

where $\varepsilon$ is the positive scalar.

\section{T-S Adaptive Observer Design}

Referring to an interpolation mechanism with the convex sum properties [7], the system (1) can be approximated by T-S fuzzy representation with multiplicative faults as follows:

$$
\begin{aligned}
\dot{x}(t)= & \sum_{i=1}^{k} \mu_{i}(\varsigma(t))\left\{\mathbf{A}_{i} x(t)+\mathbf{B}_{i} u(t)+\mathbf{D}_{i} \xi(x, t)\right. \\
& \left.+\mathbf{M}_{i} \sum_{j=1}^{q} \theta_{j}(t) h_{j}(x, u, t)\right\} \\
y(t)= & \sum_{i=1}^{k} \mu_{i}(\varsigma(t))\left\{\mathbf{C}_{i} x(t)\right\}
\end{aligned}
$$

where $\mathbf{A}_{i}, \mathbf{B}_{i}, \mathbf{D}_{i}, \mathbf{M}_{i}$, and $\mathbf{C}_{i}$ are real known matrices with appropriate dimensions. We assume that the pair $\left(\mathbf{A}_{i}, \mathbf{B}_{i}\right)$ is controllable and the pair $\left(\mathbf{A}_{i}, \mathbf{C}_{i}\right)$ is observable. Let $\mu_{i}(\zeta(t))$ be the normalized fuzzy membership functions which satisfy the properties of the sum convex.

$$
\sum_{i=1}^{k} \mu_{i}(\zeta(t))=1,0 \leq \mu_{i}(\zeta(t)) \leq 1, \quad \forall i \in[1, \ldots, k]
$$

Through the approximation of the nonlinear system (1) by augmented T-S fuzzy representation (5), we construct a multiplicative fault estimation adaptive observer as

$$
\begin{aligned}
\dot{\hat{x}}(t)= & \sum_{i=1}^{k} \mu_{i}(\varsigma(t))\left\{A_{i} \hat{x}(t)+B_{i} u(t)+G_{l, i} e_{y}(t)\right. \\
& \left.+M_{i} \sum_{j=1}^{q} \widehat{\theta}_{j}(t) h_{j}(\widehat{x}, u, t)\right\}, \\
\hat{y}(t)= & \sum_{i=1}^{k} \mu_{i}(\varsigma(t))\left\{C_{i} \hat{x}(t)\right\}, \\
\widehat{\theta}_{j}(t)= & \sigma_{j} h_{j}^{T}(\widehat{x}, u, t) \Gamma e_{y}(t), \quad j=1, \ldots, q,
\end{aligned}
$$

where $\sigma_{j}$ are positive scalars, $\widehat{x}(t)$ is the observer state, $\hat{y}(t)$ represents the observer output, $\widehat{\theta}_{j}(t)$ denotes the estimated fault magnitude, and $e_{y}(t)=y(t)-\widehat{y}(t)$ is the output estimation error. $\mathbf{G}_{l, i}$ are appropriate gain matrices, which can be obtained using LMIs as discussed later. $\Gamma$ is a design matrix representing the learning rate.
From now on, we assume that the state and fault estimation errors are defined, respectively, as $e(t)=x(t)-\widehat{x}(t)$ and $e_{\theta j}(t)=\theta_{j}(t)-\widehat{\theta}_{j}(t)$. It remains to deduce that

$$
\begin{aligned}
\dot{e}(t)= & \sum_{i=1}^{k} \mu_{i}(\varsigma(t))\left\{\left[A_{i}-G_{l, i} C_{i}\right] e(t)+D_{i} \xi(x, t)\right. \\
& \left.+M_{i} \sum_{j=1}^{q}\left[\theta_{j}(t) h_{j}(x, u, t)-\widehat{\theta}_{j}(t) h_{j}(\widehat{x}, u, t)\right]\right\},
\end{aligned}
$$

$$
\dot{e}_{\theta_{j}}(t)=\sum_{i=1}^{k} \mu_{i}(\varsigma(t))\left\{-\sigma_{j} h_{j}^{T}(\widehat{x}, u, t) \Gamma_{i} e_{y}(t)\right\} .
$$

The objective is to derive the gains of the robust adaptive observer ((7), (8), and (9)) in order to estimate multiplicative fault magnitudes.

\section{Sliding Mode Controller Design}

4.1. Adaptive Sliding Mode Controller Structure. The proposed sliding mode controller with adaptive law is assigned to provide a corrective action in order to compensate multiplicative fault effects and stabilize the nonlinear system described by T-S fuzzy representation. Before starting FTC design, we assume the following:

Assumption 3. $\operatorname{rank}\left(C_{i} B_{i}\right)=\operatorname{rank}\left(B_{i}\right), \forall i \in[1, \ldots, k]$. As the first step, one can define the sliding surface $\mathbb{S}$, when the sliding motion will take place on it, as

$$
\mathbb{S}=\left\{y(t) \in \mathbb{R}^{p}: S_{c}(t)=0\right\} .
$$

$S_{c}(t) \in \mathbb{R}^{m}$ is a linear switching function, based on the output feedback information, described as

$$
S_{c}(t)=\sum_{i=1}^{k} \mu_{i}(\zeta(t))\left\{N_{c, i} y(t)\right\}
$$

where $N_{c, i}=\left[C_{i} B_{i}\right]^{+}-h\left[I_{p}-C_{i} B_{i} C_{i} B_{i}^{+}\right]$with an arbitrary matrix $h \in \mathbb{R}^{m \times p}$. As mentioned above that $\left(A_{i}, B_{i}\right)$ is controllable, a nonlinear control input is given by

$$
u(t)=u_{l}(t)+u_{n}(t)
$$

where $u_{l}(t)$ designs the linear part which is defined as

$$
u_{l}(t)=\sum_{i=1}^{k} \sum_{j=1}^{k} \mu_{i} \mu_{j}(\varsigma(t))\left\{-K_{j} \widehat{x}(t)-F_{a, i} \widehat{f}(x, u, t)\right\}
$$

where $-F_{a, i} \widehat{f}(x, u, t)$ is designed to compensate multiplicative fault influence. It is assumed that $K_{j} \in \mathbb{R}^{m \times n}$ and $F_{a, i}=B_{i}^{+} M_{i}$.

As may be seen below, the nonlinear part $u_{n}(t)$, capable of inducing the sliding motion on the sliding surface $\mathbb{S}$, is proposed with adaptive law as 


$$
u_{n}(t)= \begin{cases}-\eta_{c}(t) \frac{S_{c}(t)}{\left\|S_{c}(t)\right\|}, & \text { if } S_{c}(t)=0, \\ 0, & \text { otherwise, }\end{cases}
$$

where $\eta_{c}(t)=\hat{\rho}_{c}+\varrho_{c}$, where $\varrho_{c}>0$ is a small constant and $\hat{\rho}_{c}$ is used to determinate $\eta_{c}(t)$ such that we will make the following adaptive term as

$$
\dot{\hat{\rho}}_{c}=\epsilon_{c}\left\|S_{c}(t)\right\|, \quad \hat{\rho}_{c}(0) \geq 0,
$$

where $\epsilon_{c}$ is the positive gain.

4.2. Reaching Condition. As mentioned earlier, it must be proven, using the nonlinear part structure of $u_{n}(t)$, that the system will be forced to reach and slide onto the corresponding sliding mode surface $\mathbb{S}$ in a finite time. In this way, we design a Lyapunov function as

$$
V_{c}(t)=\frac{1}{2} S_{c}^{T}(t) S_{c}(t)+\frac{1}{2 \epsilon_{c}}{\tilde{\rho^{\prime}}}_{c}^{2},
$$

where $\tilde{\rho}_{c}{ }_{c}=\rho_{c}-\hat{\rho}_{c}$ is the estimated error of $\rho_{c}$.

The derivative of (18) with respect to time gives

$$
\begin{gathered}
\dot{V}_{c}(t)=\sum_{i=1}^{k} \sum_{j=1}^{k} \mu_{i} \mu_{j}(\zeta(t))\left\{\left(N_{c, i} C_{i} A_{i}-K_{j}\right)\|x(t)\|\right. \\
\left.+\rho_{c}-\eta_{c}(t)\right\}\left\|S_{c}(t)\right\|-\tilde{\rho}_{c}\left\|S_{c}(t)\right\|=\sum_{i=1}^{k} \sum_{j=1}^{k} \mu_{i} \mu_{j}(\zeta(t)) \\
\cdot\left\{\left(N_{c, i} C_{i} A_{i}-K_{j}\right)\|x(t)\|-\varrho_{c}-\varepsilon_{c}\right\}\left\|S_{c}(t)\right\| .
\end{gathered}
$$

Define the subset system as

$$
\Omega_{c}=\left\{x\|x(t)\| \leq \kappa_{c}\right\} .
$$

The reachability condition, which guarantees to force the system to attain the sliding surface $\mathbb{S}$, is satisfied if the scalar $\varrho_{c}$ is chosen to satisfy $\varrho_{c}>\left(N_{c, i} C_{i} A_{i}-K_{j}\right) \kappa_{c}$ such that

$$
S_{c}^{T}(t) S_{c}(t) \leq-\varepsilon_{c}\left\|S_{c}(t)\right\| .
$$

Furthermore, the proposed sliding mode controller with adaptive law ensures the existence of an ideal sliding motion in finite time; that is, $S_{c}(t)=\dot{S}_{c}(t)=0, \forall t \geq t_{c}$.

\section{FE-Based Fault-Tolerant Control Design: A Separated Approach}

For several years, great effort has been devoted to study the FE-based FTC problem in a precise and effective way. The focus of previous studies has been on the division of this issue into separate steps:

Step 1. Conceive an observer to estimate faults and state variables.

Step 2. Conceive a controller to stabilize the closed-loop systems.

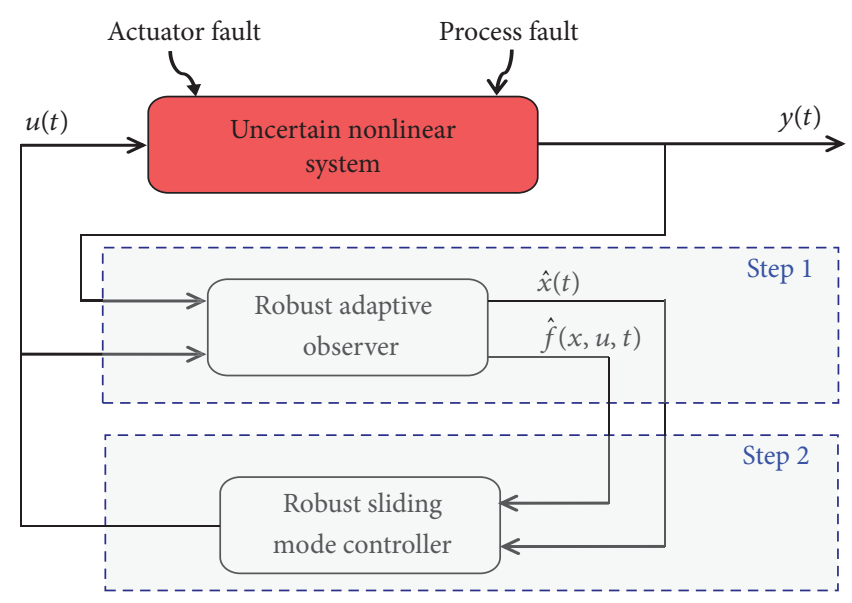

FIGURE 1: FE-based fault-tolerant control: a separated approach.

Figure 1 illustrates the separated FE-based FTC design for uncertain nonlinear systems subject to process faults and multiplicative actuator faults.

Now, we propose to design the separated FE-based FTC problem in order to compute adaptive observer gains $G_{l, i}$ and controller gains $K_{j}$ such that a robust stability of the closed-loop nonlinear system, described by the T-S form, is achieved despite the presence of multiplicative faults and uncertainties.

5.1. LMI Optimization-Based Observer Stability. Theorem 1 establishes the sufficient conditions for the stability of the observer errors $((10)$ and $(11))$ with prescribed $\mathscr{H}_{\infty}$ performances by using Lyapunov stability and LMI technique.

Theorem 1. The state estimation error is robustly stable with simultaneously maximized admissible Lipschitz constant $\gamma_{j}>0$ and minimized gain $\varsigma>0$ for the system uncertainties $\xi(x, t)$, if there exist constants, $0 \leq \lambda \leq 1, \varepsilon>0$, and $\alpha_{j}>0$, and matrices $\mathbf{P}_{e}=P_{e}^{T}>0, W_{i}$, such that the following multiobjective optimization problem has a solution:

$$
\min \left[\lambda\left(\varepsilon+\sum_{j=1}^{q} \alpha_{j}\right)+(1-\lambda) \varsigma\right],
$$

subject to the following LMI

$$
\left[\begin{array}{cccc}
\bar{\Xi}_{i}+C_{e}^{T} C_{e} & P_{e} D_{i} & P_{e} M_{i} & I_{n} \\
(*) & -\varsigma I_{l} & 0 & 0 \\
(*) & (*) & -\varepsilon I_{q} & 0 \\
(*) & (*) & (*) & -\sum_{j=1}^{q} \alpha_{j}
\end{array}\right]<0,
$$

where $\bar{\Xi}_{i}=A_{i}^{T} P_{e}+P_{e} A_{i}-W_{i} C_{i}-C_{i}{ }^{T} W_{i}{ }^{T}$. One can prove that the observer gain can be obtained from $G_{l, i}=P_{e}^{-1} W_{i}$. In addition, the T-S adaptive observer ((7), (8), and (9)) ensures that the estimated $\widehat{x}(t)$ and $\widehat{\theta}_{j}(t)$ converge to the 
nonlinear system state $x(t)$ and the multiplicative fault magnitude $\theta_{j}(t)$.

Proof. One can start by investigating the following Lyapunov function:

$$
V_{e}(t)=e^{T}(t) P_{e} e(t)+\sum_{j=1}^{q} \sigma_{j}^{-1} e_{\theta_{j}}^{T}(t) e_{\theta j}(t)>0,
$$

where $P_{e} \in \mathbb{R}^{n \times n}$ is the design Lyapunov matrix. The time derivative of $V_{e}(t)$ is handled as

$$
\begin{aligned}
\dot{V}_{e}(t)= & \dot{e}^{T}(t) P_{e} e(t)+e^{T}(t) P_{e} \dot{e}(t) \\
& +\sum_{j=1}^{q} \sigma_{j}^{-1}\left[\dot{e}_{\theta_{j}}^{T}(t) e_{\theta_{j}}(t)+e_{\theta_{j}}^{T}(t) \dot{e}_{\theta_{j}}(t)\right] .
\end{aligned}
$$

Based on state and fault estimation errors ((10) and (11)), $\dot{V}_{e}(t)$ can be written as

$$
\begin{aligned}
\dot{V}_{e}(t)= & \sum_{i=1}^{k} \mu_{i}(\zeta(t))\left\{e^{T}(t)\left[A_{l, i}^{T} P_{e}+P_{e} A_{l, i}\right] e(t)\right. \\
& +\sum_{j=1}^{q} 2 e^{T}(t) P_{e} M_{i}\left[\theta_{j}(t) h_{j}(x, u, t)-\widehat{\theta}_{j}(t) h_{j}(\hat{x}, u, t)\right] \\
& \left.+\sum_{j=1}^{q} 2 \sigma_{j}^{-1} e_{\theta_{j}}(t) \dot{\hat{\theta}}_{j}(t)+2 e^{T}(t) P_{e} D_{i} \xi(x, t)\right\}
\end{aligned}
$$

where $A_{l, i}=A_{i}-G_{l, i} C_{i}$. From this, one can conclude that

$$
\begin{aligned}
\dot{V}_{e}(t)= & \sum_{i=1}^{k} \mu_{i}(\zeta(t))\left\{e^{T}(t)\left[A_{l, i}^{T} P_{e}+P_{e} A_{l, i}\right] e(t)\right. \\
& +\sum_{j=1}^{q} 2 e^{T}(t) P_{e} M_{i}\left[\theta_{j}(t) h_{j}(x, u, t)-\theta_{j}(t) h_{j}(\widehat{x}, u, t)\right] \\
& \left.+2 e^{T}(t) P_{e} D_{i} \xi(x, t)\right\} .
\end{aligned}
$$

Referring to Lemma 1 and according to the Lipschitz condition (3), one can further derive

$$
\begin{aligned}
& 2 e^{T}(t) P_{e} M_{i}\left[\theta_{j}(t) h_{j}(x, u, t)-\theta_{j}(t) h_{j}(\hat{x}, u, t)\right] \\
& \leq \frac{1}{\varepsilon} e^{T}(t) P_{e} M_{i} M_{i}^{T} P_{e} e(t)+\varepsilon\left[h_{j}(x, u, t)-h_{j}(\widehat{x}, u, t)\right]^{T} \\
& \quad \theta_{j}^{T}(t) \theta_{j}(t)\left[h_{j}(x, u, t)-h_{j}(\hat{x}, u, t)\right] \\
& \leq \frac{1}{\varepsilon} e^{T}(t) P_{e} M_{i} M_{i}^{T} P_{e} e(t)+\varepsilon\left\|\theta_{j}(t)\right\|^{2} \\
& \quad\left\|h_{j}(x, u, t)-h_{j}(\hat{x}, u, t)\right\|^{2} \\
& \leq \frac{1}{\varepsilon} e^{T}(t) P_{e} M_{i} M_{i}^{T} P_{e} e(t)+\varepsilon \rho^{2} \gamma_{j}^{2}\|e(t)\|^{2} \\
& =\frac{1}{\varepsilon} e^{T}(t) P_{e} M_{i} M_{i}^{T} P_{e} e(t)+\varepsilon \tilde{\gamma}_{j}^{2}\|e(t)\|^{2} .
\end{aligned}
$$

where $\tilde{\gamma}_{j}=\rho \gamma_{j}$. Now, according to (28), one can derive $V_{e}(t)$ as

$$
\begin{aligned}
\dot{V}_{e}(t) & \leq \sum_{i=1}^{k} \mu_{i}(\zeta(t))\left\{e^{T}(t)\left[A_{l, i}^{T} P_{e}+P_{e} A_{l, i}\right] e(t)\right. \\
& +\frac{1}{\varepsilon} e^{T}(t) P_{e} M_{i} M_{i}^{T} P_{e} e(t)+\sum_{j=1}^{q} e^{T}(t) \varepsilon \tilde{\gamma}_{j}^{2} e(t) \\
& \left.+2 e^{T}(t) P_{e} D_{i} \xi(x, t)\right\}
\end{aligned}
$$

To attain the robustness of the proposed multiplicative fault estimation adaptive observer ((7), (8), and (9)) against system uncertainties $\xi(x, t)$, we investigate the controlled estimation error $r(t)$ as

$$
r(t)=C_{e} e(t)
$$

Consider the following worst-case performance measure:

$$
\|H\|_{\infty}=\sup _{\|\xi\|_{2} \neq 0} \frac{\|r(t)\|_{2}^{2}}{\|\xi(x, t)\|_{2}^{2}} \leq \varsigma .
$$

One can now proceed with the presence of the following variable $J_{e}(t)$ as

$$
J_{e}(t)=\dot{V}_{e}(t)+r^{T}(t) r(t)-\varsigma \xi^{T}(x, t) \xi(x, t)
$$

Obviously, one can write the above expression (32) by

$$
\begin{aligned}
J_{e}(t) & \leq \sum_{i=1}^{k} \mu_{i}(\zeta(t))\left\{e ^ { T } ( t ) \left[A_{l, i}^{T} P_{e}+P_{e} A_{l, i}+\frac{1}{\varepsilon} P_{e} M_{i} M_{i}^{T} P_{e}\right.\right. \\
& \left.+\sum_{j=1}^{q} \varepsilon \tilde{\gamma}_{j}^{2}+C_{e}^{T} C_{e}\right] e(t)+2 e^{T}(t) P_{e} D_{i} \xi(x, t) \\
& \left.-\varsigma \xi^{T}(x, t) \xi(x, t)\right\} .
\end{aligned}
$$

Let one define the following new variable as

$$
\alpha_{j}=\frac{1}{\varepsilon \tilde{\gamma}_{j}^{2}} .
$$

From now on, one can get

$$
\tilde{\gamma}_{j}=\frac{1}{\sqrt{\mathcal{E} \alpha_{j}}} .
$$

The stability of the T-S fuzzy system (5) is achieved for any fault Lipschitz nonlinear function with Lipschitz constant less than or equal to an unknown maximized constant $\tilde{\gamma}_{j}, \forall j=1, \ldots, q$. Maximization of $\tilde{\gamma}_{j}$ and minimization of $\varsigma$ can be accomplished by simultaneous minimization of $\alpha_{j}, \varepsilon$, 
and $\varsigma, \forall j=1, \ldots, q$. In this way, one can obtain a multiobjective optimization design.

As is clear from (34), one has

$$
\begin{aligned}
J_{e(t)} & \leq \sum_{i=1}^{k} \mu_{i}(\zeta(t))\left\{e ^ { T } ( t ) \left[A_{l, i}^{T} P_{e}+P_{e} A_{l, i}+\frac{1}{\varepsilon} P_{e} M_{i} M_{i}^{T} P_{e}\right.\right. \\
& \left.+\sum_{j=1}^{q} \alpha_{j}^{-1}+C_{e}^{T} C_{e}\right] e(t)+2 e^{T}(t) P_{e} D_{i} \xi(x, t) \\
& \left.-\varsigma \xi^{T}(x, t) \xi(x, t)\right\}=\Phi^{T}(t) \Delta \Phi(t),
\end{aligned}
$$

where

$$
\begin{aligned}
\Phi(t) & =\left[\begin{array}{c}
e(t) \\
\xi(x, t)
\end{array}\right], \\
\Delta & =\sum_{i=1}^{k} \mu_{i}(\zeta(t))\left[\begin{array}{cc}
\Xi_{i j}+C_{e}^{T} C_{e} & P_{e} D_{i} \\
D_{i}^{T} P_{e} & -\varsigma
\end{array}\right], \\
\Xi_{i j} & =A_{l, i}^{T} P_{e}+P_{e} A_{l, i}+\frac{1}{\varepsilon} P_{e} M_{i} M_{i}^{T} P_{e}+\sum_{j=1}^{q} \alpha_{j}^{-1} .
\end{aligned}
$$

One can conclude that if $\Delta<0$, it implies that $J_{e}(t)<0$.

According to the Schur complement, the previous inequality is satisfied if and only if we checked for the presence of this relation:

$$
\sum_{i=1}^{k} \mu_{i}(\zeta(t)) \Omega_{i j}<0
$$

where $\Omega_{i j}$ are the same as those in (23).

In this way, the state estimation error is asymptotically stable with the attenuation level $\varsigma$ as follows:

$$
\|r(t)\|_{2}^{2} \leq \varsigma\|\xi(x, t)\|_{2}^{2} .
$$

It is obvious that $e(t)=x(t)-\widehat{x}(t) \rightarrow 0$. Due to the fault, nonlinear function $h_{j}(x, u, t)$ satisfies the Lipschitz condition; the T-S multiplicative fault estimation adaptive observer $\left((7),(8)\right.$, and (9)) ensures that $e_{\theta_{j}}(t) \rightarrow 0$. From this, one can deduce that, according to (9), the estimation of multiplicative fault magnitude $\theta_{j}(t)$, for the uncertain nonlinear system (1) described by T-S fuzzy structure (5), can be achieved.

This completes the proof.

5.2. LMI Optimization-Based Closed-Loop Stability. Once the sliding mode is obtained, we consider to analyze the stability of the closed-loop T-S fuzzy system. Let the equivalent control $u_{\text {eq }}(t)$, such that $\dot{S}_{c}(t)$ is equal to zero, be

$$
\left.u_{\mathrm{eq}}(t)=\sum_{i=1}^{k} \mu_{i}(\zeta(t))\left\{-N_{c, i} C_{i}\left[A_{i} x(t)+D_{i} \xi(x, t)\right]\right\}+u_{l}(t)\right\} .
$$

The dynamic of the closed-loop system with the equivalent control law (40) takes the form

$$
\begin{aligned}
& \dot{x}(t)=\sum_{i=1}^{k} \sum_{j=1}^{k} \mu_{i} \mu_{j}(\zeta(t))\left\{\left(\Theta_{i} A_{i}-B_{i} K_{j}\right) x(t)+\bar{B}_{i, j} \phi(t)\right\} \\
& y(t)=\sum_{i=1}^{k} \mu_{i}(\zeta(t))\left\{C_{i} x(t)\right\}
\end{aligned}
$$

where $\bar{B}_{i, j}=\left[B_{i} K_{j} M_{i} \Theta_{i} D_{i}\right], \Theta_{i}=I_{n}-B_{i} N_{c, i} C_{i}$, and $\phi(t)=$ $\left[\begin{array}{lll}e^{T}(t) & e_{f}^{T}(x, u, t) & \xi^{T}(x, t)\end{array}\right]^{T}$, where $e_{f}(x, u, t)=f(x, u, t)$ $-\widehat{f}(x, u, t)$.

The objective now is to develop a sufficient condition to achieve the stability of the closed-loop T-S fuzzy system ((41) and (42)) on the sliding surface $S$ despite the occurrence of multiplicative faults and the presence of uncertainties.

Theorem 2. The closed-loop T-S fuzzy system ((41) and (42)) is robustly stable with the $H_{\infty}$ attenuation level $\varsigma_{s}>0$, if there exist the matrices $\overline{\mathbf{P}}_{x}=\bar{P}_{x}^{T}>0, \mathbf{Q}_{j}$, and $\mathbf{Y}=Y^{T}$, such that

$$
\min \left[\varsigma_{s}\right]
$$

satisfying the following LMI constraints:

$$
\Delta_{\text {SepaFTC }}=\left[\begin{array}{ccccc}
\Upsilon_{i, j} & B_{i} Q_{j} & M_{i} & \Theta_{i} D_{i} & \bar{P}_{x} C_{i}^{T} \\
(*) & -2 Y+\varsigma_{s} I_{n} & 0 & 0 & 0 \\
(*) & (*) & -\varsigma_{s} I_{q} & 0 & 0 \\
(*) & (*) & (*) & -\varsigma_{s} I_{l} & 0 \\
(*) & (*) & (*) & (*) & -I_{p}
\end{array}\right]<0
$$

where $\Upsilon i, j=\bar{P}_{x} A_{i}^{T} \Theta_{i}^{T}+\Theta_{i} A_{i} \bar{P}_{x}-B_{i} Q_{j}-Q_{j}^{T} B_{i}^{T}, \quad Y=\varsigma_{s} \bar{P}_{x}$, and $K_{j}=Q_{j} \bar{P}_{x}^{-1}$.

Proof. Consider the following Lyapunov function for the closed-loop system as

$$
V_{x}(t)=x^{T}(t) P_{x} x(t)
$$

where $P_{x} \in \mathbb{R}^{n \times n}$ is the symmetric positive definite matrix. The time derivative of $V_{x}(t)$ is handled as

$$
\begin{aligned}
\dot{V}_{x}(t)= & \sum_{i=1}^{k} \sum_{j=1}^{k} \mu_{i} \mu_{j}(\zeta(t))\left\{x ^ { T } ( t ) \left[\left(\Theta_{i} A_{i}-B_{i} K_{j}\right)^{T} P_{x}\right.\right. \\
& \left.\left.+P_{x}\left(\Theta_{i} A_{i}-B_{i} K_{j}\right)\right] x(t)+2 x^{T}(t) P_{x} \bar{B}_{i, j} \phi(t)\right\} .
\end{aligned}
$$


To achieve the robustness with $H_{\infty}$ performance of the closed-loop T-S fuzzy system $((41)$ and $(42))$ to $\phi(t)$, the following inequality must then hold:

$$
J_{x}(t)=\dot{V}_{x}(t)+y^{T}(t) y(t)-\varsigma_{s} \phi^{T}(t) \phi(t)<0 .
$$

Insertion of (46) in (47) yields

$$
\begin{aligned}
J_{x}(t)= & \sum_{i=1}^{k} \sum_{j=1}^{k} \mu_{i} \mu_{j}(\zeta(t))\left\{x ^ { T } ( t ) \left[\left(\Theta_{i} A_{i}-B_{i} K_{j}\right)^{T} P_{x}\right.\right. \\
& \left.+P_{x}\left(\Theta_{i} A_{i}-B_{i} K_{j}\right)+C_{i}^{T} C_{i}\right] x(t)+2 x^{T}(t) P_{x} \bar{B}_{i, j} \phi(t) \\
& \left.-\varsigma_{s} \phi^{T}(t) \phi(t)\right\} .
\end{aligned}
$$

Consequently (in the matrix form), it remains to prove that $J_{x}(t)<0$, if

$$
\left[\begin{array}{cc}
\psi_{i, j} & P_{x} \bar{B}_{i, j} \\
\bar{B}_{i, j}^{T} P_{x} & -\varsigma_{s} I
\end{array}\right]<0,
$$

where $\quad \psi_{i, j}=\left(\Theta_{i} A_{i}-B_{i} K_{j}\right)^{T} P_{x}+P_{x}\left(\Theta_{i} A_{i}-B_{i} K_{j}\right)+C_{i}^{T} C_{i}$. Using the Schur complement, the relation (49) can be reformulated as

$$
\prod_{i, j}=\left[\begin{array}{ccccc}
\sum_{i, j} & P_{x} B_{i} K_{j} & P_{x} M_{i} & P_{x} \Theta_{i} D_{i} & C_{i}^{T} \\
(*) & -\varsigma_{s} I_{n} & 0 & 0 & 0 \\
(*) & (*) & -\varsigma_{s} I_{q} & 0 & 0 \\
(*) & (*) & (*) & -\varsigma_{s} I_{l} & 0 \\
(*) & (*) & (*) & (*) & -I_{p}
\end{array}\right]<0,
$$

where $\sum_{i, j}=\Theta_{i}^{T} A_{i}^{T} P_{x}+P_{x} \Theta_{i} A_{i}-K_{j}^{T} B_{i}^{T} P_{x}-P_{x} B_{i} K_{j}$.

Inequality (50) contains several nonlinear terms. One can design in the next step to formulate this as an LMI problem. To effect the necessary change of variables, one will define the following matrix $\mathbf{X}$ with the special diagonal structure as $\mathbf{X}=\operatorname{diag}\left\{P_{x}^{-1}, P_{x}^{-1}, I_{q}, I_{l}, I_{p}\right\}$. Then, $X \times \Pi_{i, j} \times X^{T}<0$ is true, and it is obvious that

$$
\left[\begin{array}{ccccc}
\Upsilon_{i, j} & B_{i} Q_{j} & M_{i} & \Theta_{i} D_{i} & \bar{P}_{x} C_{i}^{T} \\
(*) & -\varsigma_{s} \bar{P}_{x} \bar{P}_{x} & 0 & 0 & 0 \\
(*) & (*) & -\varsigma_{s} I_{q} & 0 & 0 \\
(*) & (*) & (*) & -\varsigma_{s} I_{l} & 0 \\
(*) & (*) & (*) & (*) & -I_{p}
\end{array}\right]<0,
$$

where $\Upsilon_{i, j}=\bar{P}_{x} A_{i}^{T} \Theta_{i}^{T}+\Theta_{i} A_{i} \bar{P}_{x}-B_{i} Q_{j}-Q_{i}^{T} B_{i}^{T}, \bar{P}_{x}=P_{x}^{-1}$, and $Q_{j}=K_{j} P_{x}^{-1}$. According to Lemma 1 , it is evident to check the presence of the following relation

$$
\bar{P}_{x}+\bar{P}_{x} \leq \bar{P}_{x} \bar{P}_{x}+I_{n} .
$$

Obviously, (52) is true for $\varsigma_{c}>0$ as

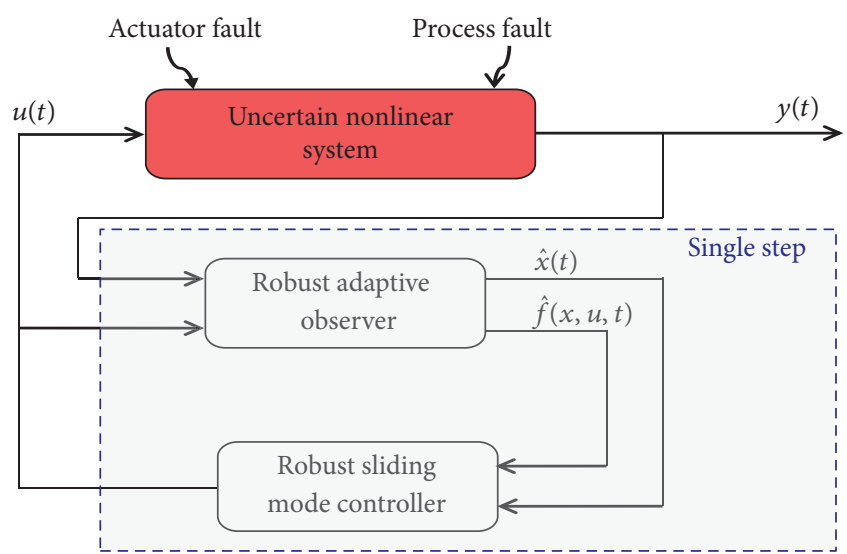

Figure 2: FE-based fault-tolerant control: an integrated approach.

$$
-\varsigma_{s} \bar{P}_{x} \bar{P}_{x} \leq-2 \varsigma_{s} \bar{P}_{x}+\varsigma_{s} I_{n} .
$$

After some manipulations, one can get

$$
\sum_{i=1}^{k} \sum_{j=1}^{k} \mu_{i} \mu_{j}(\zeta(t)) \Delta_{\text {SepaFTC }}<0,
$$

where $\Delta_{\text {SepaFTC }}$ has the same structure with (44). Clearly, a stability proof of the closed-loop T-S fuzzy system ((41) and (42)) is required with respect to the $H_{\infty}$ performance level $\varsigma_{s}$.

This completes the proof.

\section{FE-Based Fault-Tolerant Control Design: An Integrated Approach}

Several publications have appeared in recent years documenting FE-based FTC design with a single step in order to achieve an optimal robustness interaction between observer and controller models. Figure 2 illustrates the integrated FE-based FTC design for uncertain nonlinear systems subject to process fault and multiplicative actuator fault.

In this section, we explore the possibility of the integrated FE-based FTC design to compute, in a single step, adaptive observer gains $G_{l, i}$ and controller gains $K_{j}$ in the sense that it ameliorates the robustness of the closed-loop nonlinear system despite the presence of multiplicative faults and uncertainties. Combining (10), (11), and (41) gives the following augmented closed-loop system, including fault estimation with fault compensation control, expressed as

$$
\dot{x}(t)=\sum_{i=1}^{k} \sum_{j=1}^{k} \mu_{i} \mu_{j}(\zeta(t))\left\{\left(\Theta_{i} A_{i}-B_{i} K_{j}\right) x(t)+\bar{B}_{i, j} \phi(t)\right\},
$$

$$
\begin{aligned}
\dot{e}(t)= & \sum_{i=1}^{k} \mu_{i}(\zeta(t))\left\{\left[A_{i}-G_{l, i} C_{i}\right] e(t)+\bar{D}_{i}^{1} \phi(t)\right. \\
& \left.+M_{i} \sum_{j=1}^{q}\left[\theta_{j}(t) h_{j}(x, u, t)-\widehat{\theta}(t) h_{j}(\widehat{x}, u, t)\right]\right\},
\end{aligned}
$$




$$
\begin{gathered}
\dot{e}_{\theta_{j}}(t)=\sum_{i=1}^{k} \mu_{i}(\zeta(t))\left\{-\sigma_{j} h_{j}^{T}(\widehat{x}, u, t) \Gamma_{i} e_{y}(t)\right\}, \\
y_{L}(t)=\sum_{i=1}^{k} \mu_{i}(\zeta(t))\left\{C_{L, i} x(t)+C_{e, i} e(t)\right\},
\end{gathered}
$$

where $\bar{B}_{i, j}=\left[\begin{array}{lll}B_{i} K_{j} & M_{i} & \Theta_{i} D_{i}\end{array}\right], \bar{D}_{i}^{1}=\left[\begin{array}{lll}0 & 0 & D_{i}\end{array}\right], \Theta_{i}=I_{n}$ $-B_{i} N_{c, i} C_{i}$, and $\phi(t)-\left[\begin{array}{lll}e(t)^{T} & e_{f}^{T}(x, u, t) & \xi^{T}(x, t)\end{array}\right]^{T} .$.

Theorem 3. Under the sliding mode input structure (14), the closed-loop T-S fuzzy system ((55), (56), (57), and (58)) is robustly stable with both maximized admissible Lipschitz constant $\gamma_{e, j}>0$ and minimized gain $\left\|H_{y L \phi}\right\|_{\infty}<\varsigma_{c}$, if there exist constants, $0 \leq \lambda_{c} \leq 1, \varepsilon_{e}>0$, and $\alpha_{e, j}>0$, and matrices $\mathbf{P}_{e}=P_{e}^{T}>0, \overline{\mathbf{P}}_{x}=\bar{P}_{x}^{T}>0, \mathbf{W}_{i}, \mathbf{Q}_{j}$, and $\mathbf{Y}=Y^{T}$, such that the multiobjective LMI optimization problem admits a solution as

$$
\min \left[\lambda_{c}\left(\varepsilon_{e}+\sum_{j=1}^{q} \alpha_{e, j}\right)+\left(1-\lambda_{c}\right) \varsigma_{c}\right],
$$

subject to $\quad \bar{\Xi}_{i j}=\left[\begin{array}{cc}\bar{\Xi}_{11, i j} & \bar{\Xi}_{12, i j} \\ (*) & \bar{\Xi}_{22, i j}\end{array}\right]<0$,

where

$$
\begin{aligned}
& \Xi_{11, i j}=\left[\begin{array}{cc}
\Xi_{11, i j} & 0 \\
(*) & \Xi_{22, i}
\end{array}\right] \text {, } \\
& \Xi_{11, i j}=\operatorname{He}\left(\Theta_{i} A_{i} \bar{P}_{x}\right)-H e\left(B_{i} Q_{j}\right), \\
& \Xi_{22, i}=H e\left(P_{e} A_{i}\right)-H e\left(W_{i} C_{i}\right)+C_{e, i}^{T} C_{e, i} W_{i}=P_{e} G_{l, i} \\
& \bar{\Xi}_{12, i j}=\left[\begin{array}{cccccc}
B_{i} Q_{j} & M_{i} & \Theta_{i} D_{i} & \bar{P}_{x} C_{L, i}^{T} & 0 & 0 \\
0 & 0 & P_{e} D_{i} & 0 & P_{e} M_{i} & I_{n}
\end{array}\right] \text {, } \\
& \bar{\Xi}_{22, i j}=-\operatorname{diag}\left[2 Y-\varsigma_{c} I_{n}, \varsigma_{c} I_{q}, \varsigma_{c} I_{l}, I_{p}, \varepsilon_{e} I_{q}, \sum_{j=1}^{q} \alpha_{e, j}^{-1}\right] \text {, } \\
& Y=\varsigma_{c} \bar{P}_{x}, \bar{P}_{x}=P_{x}^{-1}, Q_{j}=K_{j} P_{x}^{-1} .
\end{aligned}
$$

The gain matrices of the adaptive sliding mode controller $K_{j}$ and observer $G_{l, i}$ are given by

$$
\begin{aligned}
K_{j} & =Q_{j} \bar{P}_{x}^{-1}, \\
G_{l, i} & =P_{e}^{-1} W_{i} .
\end{aligned}
$$

Proof. Stability analysis: In order to assure the stability of the augmented closed-loop system ((55), (56), (57), and (58)), one can start by investigating the following Lyapunov function as

$$
V(t)=V_{e}(t)+V_{x}(t)
$$

where $\quad V_{e}(t)=e^{T}(t) P_{e} e(t)+\sum_{j=1}^{q} \sigma_{j}^{-1} e_{\theta_{j}}^{T}(t) e_{\theta_{j}}(t)>0 \quad$ and $V_{x}(t)=x^{T}(t) P_{x} x(t)>0$, and $P_{x} \in \mathbb{R}^{n \times n}$ is the symmetric positive definite matrix.
As first, one can proceed analogously to Theorem 1 . Hence, the time derivative of $V_{e}(t)$ is bounded as

$$
\begin{aligned}
\dot{V}_{e}(t) & \leq \sum_{i=1}^{k} \mu_{i}(\zeta(t))\left\{e ^ { T } ( t ) \left[A_{l, i}^{T} P_{e}+P_{e} A_{l, i}+\frac{1}{\varepsilon_{e}} P_{e} M_{i} M_{i}^{T} P_{e}\right.\right. \\
& \left.\left.+\sum_{j=1}^{q} \alpha_{e, j}^{-1}\right] e(t)+2 e^{T}(t) P_{e} \bar{D}_{i}^{1} \phi(t)\right\} .
\end{aligned}
$$

On the other hand, similar to Theorem 2 and by taking into account the closed-loop T-S fuzzy system (55), the time derivative of $V_{x}(t)$ is expressed as

$$
\begin{aligned}
\dot{V}_{x}(t)= & \sum_{i=1}^{k} \sum_{j=1}^{k} \mu_{i} \mu_{j}(\zeta(t))\left\{x ^ { T } ( t ) \left[\left(\Theta_{i} A_{i}-B_{i} K_{j}\right)^{T} P_{x}\right.\right. \\
& \left.\left.+P_{x}\left(\Theta_{i} A_{i}-B_{i} K_{j}\right)\right] x(t)+2 x^{T}(t) P_{x} \bar{B}_{i j} \phi(t)\right\} .
\end{aligned}
$$

Robust performance index: Let

$$
J(t)=\dot{V}(t)+y_{L}^{T}(t) y_{L}(t)-\varsigma_{c} \phi^{T}(t) \phi(t)<0 .
$$

The inequality (65), after substituting (63) and (64), becomes

$$
\begin{aligned}
J(t) & \leq \sum_{i=1}^{k} \sum_{j=1}^{k} \mu_{i} \mu_{j}(\zeta(t))\left\{x ^ { T } ( t ) \left[\left(B_{i j}^{1}\right)^{T} P_{x}\right.\right. \\
& \left.+P_{x} B_{i j}^{1}+C_{L, i}^{T} C_{L, i}\right] x(t)+e^{T}(t)\left[A_{l, i}^{T} P_{e}+P_{e} A_{l, i}\right. \\
& \left.+\frac{1}{\varepsilon_{e}} P_{e} M_{i} M_{i}^{T} P_{e}+\sum_{j=1}^{q} \alpha_{e, j}^{-1}+C_{e, i}^{T} C_{e, i}\right] e(t) \\
& \left.+2 x^{T}(t) P_{x} \bar{B}_{i j} \phi(t)+2 e^{T}(t) P_{e} \bar{D}_{i}^{1} \phi(t)-\varsigma_{c} \phi^{T}(t) \phi(t)\right\} .
\end{aligned}
$$

where $B_{i j}^{1}=\Theta_{i} A_{i}-B_{i} K_{j}$. Equivalently, in the matrix form, one can obtain the following expression as

$$
J(t) \leq \sum_{i=1}^{k} \sum_{j=1}^{k} \mu_{i} \mu_{j}(\zeta(t))\left\{\chi^{T}(t) \Gamma_{i j} \chi(t)\right\},
$$

where $\chi(t)=\left[\begin{array}{lll}x^{T}(t) & e^{T}(t) & \phi^{T}(t)\end{array}\right]^{T}$. The variable $\Gamma_{i j}$ is defined as

$$
\Gamma_{i j}=\left[\begin{array}{ccccc}
\Gamma_{11, i j} & 0 & P_{x} B_{i} K_{j} & P_{x} M_{i} & P_{x} \Theta_{i} D_{i} \\
(*) & \Gamma_{22, i} & 0 & 0 & P_{e} D_{i} \\
(*) & (*) & -\varsigma_{c} I_{n} & 0 & 0 \\
(*) & (*) & (*) & -\varsigma_{c} I_{q} & 0 \\
(*) & (*) & (*) & (*) & -\varsigma_{c} I_{l}
\end{array}\right],
$$


such that

$$
\begin{gathered}
\Gamma_{11, i j}=H e\left(\left[\Theta_{i} A_{i}-B_{i} K_{j}\right]^{T} P_{x}\right)+C_{L, i}^{T} C_{L, i}, \\
\Gamma_{22, i}=H e\left(A_{l, i}^{T} P_{e}\right)+\frac{1}{\varepsilon_{e}} P_{e} M_{i} M_{i}^{T} P_{e}+\sum_{j=1}^{q} \alpha_{e, j}^{-1}+C_{e, i}^{T} C_{e, i} .
\end{gathered}
$$

To effect the necessary change of variables, one will make the following matrix $\mathbf{X}$ with the special diagonal structure as $\mathbf{X}=\operatorname{diag}\left\{P_{x}^{-1}, I_{n}, P_{x}^{-1}, I_{q}, I_{l}\right\}$. After pre- and postmultiplying by $X$ and its transpose in $\Gamma_{i j}$, then it is obvious that

$$
\bar{\Gamma}_{i j}=\left[\begin{array}{ccccc}
\bar{\Gamma}_{11, i j} & 0 & B_{i} Q_{j} & M_{i} & \Theta_{i} D_{i} \\
(*) & \bar{\Gamma}_{22, i} & 0 & 0 & P_{e} D_{i} \\
(*) & (*) & -\varsigma_{c} \bar{P}_{x} \bar{P}_{x} & 0 & 0 \\
(*) & (*) & (*) & -\varsigma_{c} I_{q} & 0 \\
(*) & (*) & (*) & (*) & -\varsigma_{c} I_{l}
\end{array}\right],
$$

where

$$
\begin{aligned}
& \bar{\Gamma}_{11, i j}= H e\left(\Theta_{i} A_{i} \bar{P}_{x}\right)-\operatorname{He}\left(B_{i} Q_{j}\right)+\bar{P}_{x} C_{L, i}^{T} C_{L, i} \bar{P}_{x}, \\
& \bar{\Gamma}_{22, i}= H e\left(P_{e} A_{i}\right)+H e\left(W_{i} C_{i}\right)+\frac{1}{\varepsilon_{e}} P_{e} M_{i} M_{i}^{T} P_{e} \\
&+\sum_{j=1}^{q} \alpha_{e, j}^{-1}+C_{e, i}^{T} C_{e, i}, \\
& \bar{P}_{x}=P_{x}^{-1}, Q_{j}=K_{j} \bar{P}_{x}^{-1}, W_{i}=P_{e} G_{l, i} .
\end{aligned}
$$

After simple manipulation by using Lemma 1 , it is evident to obtain the relation (59). From this, one can conclude that the augmented closed-loop T-S fuzzy system ((55), (56), (57), and (58)) is robustly stable against $e(t), e_{f}(x, u, t)$, and $\xi(x, t)$ with respect to the $H_{\infty}$ performance level $\varsigma_{c}$.

This completes the proof.

\section{Illustrative Example}

In the present section, the design of the separated and integrated sliding mode fault-tolerant control based on adaptive observer information requirement is performed by considering the nonlinear model of a single-link flexible-joint robot arm taken from [13]. Firstly, let us consider the nonlinear model without faults defined by

$$
\begin{aligned}
\dot{\theta}_{\mathrm{m}} & =\omega_{\mathrm{m}}, \\
\dot{\omega}_{\mathrm{m}} & =\frac{k}{J_{\mathrm{m}}}\left(\theta_{1}-\theta_{\mathrm{m}}\right)-\frac{B_{v}}{J_{\mathrm{m}}} \omega_{\mathrm{m}}+\frac{K_{\tau}}{J_{\mathrm{m}}} u(t), \\
\dot{\theta}_{\mathrm{l}} & =\omega_{\mathrm{l}}, \\
\dot{\omega}_{1} & =\frac{k}{J_{1}}\left(\theta_{1}-\theta_{\mathrm{m}}\right)-\frac{m g h}{J_{\mathrm{l}}} \sin \left(\theta_{\mathrm{l}}\right),
\end{aligned}
$$

TABLE 1: Nonlinear system parameters.

\begin{tabular}{lcc}
\hline System parameters & Values & Units \\
\hline Motor inertia $J_{\mathrm{m}}$ & $3.7 \times 10^{-3}$ & $\mathrm{~kg} \mathrm{~m}^{2}$ \\
Link inertia $J_{1}$ & $9.3 \times 10^{-3}$ & $\mathrm{~kg} \mathrm{~m}^{2}$ \\
Pointer mass $m$ & 0.21 & $\mathrm{~kg}$ \\
Link length $h$ & 0.15 & $\mathrm{~m}$ \\
Torsional spring $k$ & 0.18 & $\mathrm{Nm} / \mathrm{rad}$ \\
Viscous friction $B_{v}$ & $4.6 \times 10^{-3}$ & $\mathrm{M}$ \\
Amplifier gain $K_{\tau}$ & $8 \times 10^{-2}$ & $\mathrm{Nm} / \mathrm{V}$ \\
\hline
\end{tabular}

where $\theta_{\mathrm{m}}$ and $\omega_{\mathrm{m}}$ are the position and angular velocity of the DC motor, respectively, and $\theta_{1}$ and $\omega_{1}$ represent the position and angular velocity of the link. The values of the parameters are given in Table 1.

We choose that $x_{1}=\theta_{\mathrm{m}}, x_{2}=\omega_{\mathrm{m}}, x_{3}=\theta_{1}$, and $x_{4}=\omega_{1}$.

The flexible-joint robot arm system is described in the nonlinear form as follows:

$$
\dot{x}(t)=A x(t)+B u(t)+\Gamma(x, t)+M f(x, u, t)+D \xi(x, t),
$$

$$
y(t)=C x(t)
$$

with

$$
\begin{aligned}
& A=\left[\begin{array}{cccc}
0 & 1 & 0 & 0 \\
-48.6 & -1.25 & 48.6 & 0 \\
0 & 0 & 0 & 1 \\
19.5 & 0 & -19.5 & 0
\end{array}\right], \\
& B=M=\left[\begin{array}{c}
0 \\
21.62 \\
0 \\
0
\end{array}\right] \text {, } \\
& D=\left[\begin{array}{c}
0 \\
0.25 \\
0 \\
0
\end{array}\right] \text {, } \\
& C=\left[\begin{array}{llll}
1 & 0 & 0 & 0 \\
0 & 1 & 0 & 0 \\
0 & 0 & 1 & 0
\end{array}\right], \\
& \Gamma(x, u, t)=\left[\begin{array}{c}
0 \\
21.62 u(t) \\
0 \\
3.33 \sin \left(x_{3}(t)\right)
\end{array}\right] \text {, }
\end{aligned}
$$




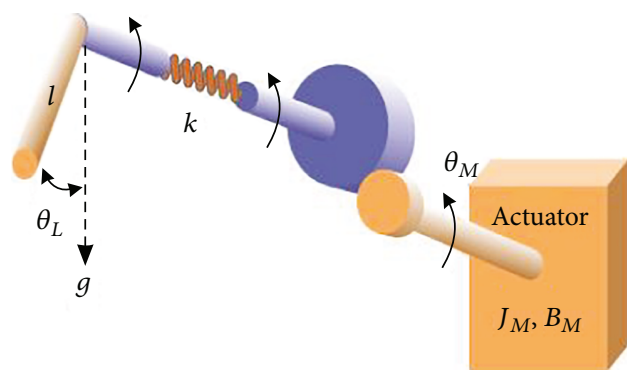

FIGURE 3: Schematic diagram of a single-link flexible-joint robot arm.

TABLE 2: LMI optimization gains.

\begin{tabular}{lccc}
\hline & \multirow{2}{*}{ Integrated FTC } & \multicolumn{2}{c}{ Separated FTC } \\
& & Observer & Controller \\
\hline $\begin{array}{l}\text { Uncertainty } \\
\text { attenuation level }\end{array}$ & $\varsigma_{c}=0.1588$ & $\varsigma=0.0612$ & $\varsigma_{s}=0.7337$ \\
$\begin{array}{l}\text { Admissible } \\
\text { Lipschitz constant }\end{array}$ & $\gamma_{1}=\gamma_{2}=0.7916$ & $\gamma_{e, 1}=\gamma_{e, 2}=0.3911$ \\
\hline
\end{tabular}

where $\Gamma(x, u, t)$ encapsulates the nonlinearities present in the DC motor. The schematic diagram of a single-link flexible-joint robot arm is shown in Figure 3.

To evaluate the performances of the proposed FE-based adaptive sliding mode fault-tolerant control, we consider the presence of two types of multiplicative faults affecting the considered nonlinear system, which are described in the following.

(1) Actuator gain fault (loss of effectiveness): multiplicative fault occurs in the actuator which is defined as a partial loss of effectiveness. We suppose that fault magnitude $\theta_{1}(t)$ has the following structure:

$$
\theta_{1}(t)=\left\{\begin{array}{c}
0, \quad t<5 \mathrm{sec} \\
0.8, \quad 5 \mathrm{sec} \leq t<20 \mathrm{sec}, \\
0, \quad t>15 \mathrm{sec} .
\end{array}\right.
$$

The fault function $h_{1}(x, u, t)$ is expressed as

$$
h_{1}(x, u, t)=\left[\begin{array}{llll}
0 & u(t) & 0 & 0
\end{array}\right]^{T} .
$$

In this case, the first multiplicative fault is modeled as

$$
f_{1}(x, u, t)=\left[\begin{array}{llll}
0 & 3 \theta_{1}(t) u(t) & 0 & 0
\end{array}\right]^{T} .
$$

(2) Abnormal friction subject to process fault: an abnormal friction appears in the DC motor where it leads to parameter changes in the nonlinear system state matrix. This multiplicative process fault has the following special structure:

$$
f_{2}(x, u, t)=\left[\begin{array}{llll}
0 & -5 \theta_{2}(t) \omega_{\mathrm{m}}(t) & 0 & 0
\end{array}\right]^{T},
$$

which corresponds to the structure function handled as

$$
h_{2}(x, u, t)=\left[\begin{array}{llll}
0 & -5 \omega_{\mathrm{m}}(t) & 0 & 0
\end{array}\right]^{T} .
$$

We suppose that the viscous friction constant $B_{v}$ increases by $80 \%$ at $t=2.5 \mathrm{sec}$, that is, $\theta_{2}(t)=0$ at $t<2.5$ sec and $\theta_{2}(t)=0.8$ at $t \geq 2.5 \mathrm{sec}$.
The flexible-joint robot arm system can be formulated in the T-S representation (5), where $k=2$, with the system matrices:

$$
\mathbf{A}_{1}=\left[\begin{array}{cccc}
0 & 1 & 0 & 0 \\
-48.6 & -1.25 & 48.6 & 0 \\
0 & 0 & 0 & 1 \\
19.5 & 0 & -22.83 & 0
\end{array}\right] \text {, }
$$

$$
\mathbf{B}_{1}=\mathbf{M}_{1}=\left[\begin{array}{c}
0 \\
21.62 \\
0 \\
0
\end{array}\right] \text {, }
$$

$$
\mathbf{A}_{2}=\left[\begin{array}{cccc}
0 & 1 & 0 & 0 \\
-48.6 & -1.24 & 48.6 & 0 \\
0 & 0 & 0 & 1 \\
19.5 & 0 & -18.77 & 0
\end{array}\right] \text {, }
$$

$$
\mathbf{B}_{2}=\mathbf{M}_{2}=\left[\begin{array}{c}
0 \\
21.62 \\
0 \\
0
\end{array}\right]
$$

$$
\begin{aligned}
& \mathbf{D}_{1}=\mathbf{D}_{2}=\left[\begin{array}{c}
0 \\
0.25 \\
0 \\
0
\end{array}\right], \\
& \mathbf{C}_{1}=\mathbf{C}_{2}=\left[\begin{array}{llll}
1 & 0 & 0 & 0 \\
0 & 1 & 0 & 0 \\
0 & 0 & 1 & 0
\end{array}\right] .
\end{aligned}
$$

The parameters $\mu_{i}(x(t))$ are given by

$$
\begin{aligned}
& \mu_{1}(x(t))=\frac{\vartheta(t)+0.21}{1.21}, \\
& \mu_{2}(x(t))=\frac{1-\vartheta(t)}{1.21},
\end{aligned}
$$

where $\vartheta(t)=\sin \left(x_{3}(t)\right) / x_{3}(t)$.

Comparative simulations are given using the separated and integrated multiplicative FE-based FTC design with the same system parameters and initial conditions.

\subsection{Separated Multiplicative FE-Based FTC Design}

7.1.1. First Step: Adaptive Observer Design. The design parameters were chosen as $\lambda=0.9$ and $H=I_{4 \times 4}$. By solving Theorem 1 with the MATLAB LMI Toolbox, the adaptive observer ((7), (8), and (9)) design is achieved as 


$$
\begin{gathered}
G_{l, 1}=10^{4} \times\left[\begin{array}{ccc}
0.0001 & -0.0021 & -0.0088 \\
4.0422 & 0.1157 & 3.8222 \\
0.0117 & -0.0027 & 0.0001 \\
0.0077 & -0.0013 & -0.0021
\end{array}\right], \\
G_{l, 2}=10^{4} \times\left[\begin{array}{ccc}
0.0006 & 0.0023 & -0.0050 \\
-2.5615 & 1.1566 & -0.6657 \\
0.0066 & 0.0005 & 0.0015 \\
0.0228 & 0.0003 & -0.0170
\end{array}\right],
\end{gathered}
$$

where we find that

$$
P_{e}=\left[\begin{array}{cccc}
4.1673 & 0 & 0 & 0 \\
0 & 0.0022 & 0 & 0 \\
0 & 0 & 3.7929 & -2.0669 \\
0 & 0 & -2.0669 & 4.1673
\end{array}\right] .
$$

7.1.2. Second Step: Sliding Mode Controller Design. From Theorem 2, the sliding mode controller gains (14) are described as

$$
K_{1}=K_{2}=\left[\begin{array}{llll}
101.5736 & 11.6305 & -34.4968 & 11.4298
\end{array}\right],
$$

such that

$$
P_{x}=\left[\begin{array}{cccc}
0.2650 & -0.9467 & 0.2119 & -0.8697 \\
-0.9467 & 12.3589 & 0.0313 & 0.0634 \\
0.2119 & 0.0313 & 0.4505 & -0.5907 \\
-0.8697 & 0.0634 & -0.5907 & 6.2204
\end{array}\right] .
$$

7.2. Integrated Multiplicative FE-Based FTC Design. By solving the LMI conditions given in Theorem 3, using the "mincx" function of the MATLAB LMI toolbox, the matrix gains of the adaptive observer ((7), (8), and (9)) and the sliding mode controller (14) are computed in a single step as

$$
\begin{aligned}
& G_{l, 1}=\left[\begin{array}{ccc}
0.5001 & 0.4705 & -4.4445 \\
386.9460 & 475.4862 & 24.2993 \\
4.4446 & 0.0295 & 0.9559 \\
21.2220 & 0.0114 & -21: 4797
\end{array}\right] \text {, } \\
& G_{l, 2}=\left[\begin{array}{ccc}
0.5001 & 0.4705 & -4.4445 \\
386.9496 & 475.4862 & 24.3004 \\
4.4446 & 0.0295 & 0.9559 \\
21.2220 & 0.0114 & -17.4097
\end{array}\right] \text {, } \\
& K_{1}=K_{2}=\left[\begin{array}{llll}
102.6561 & 11.4223 & -35.6482 & 12.0718
\end{array}\right] \text {, }
\end{aligned}
$$

where we find that

$$
\begin{gathered}
P_{e}=10^{4} \times\left[\begin{array}{rccc}
0.9035 & 0 & 0 & 0 \\
0 & 0.0011 & 0 & 0 \\
0 & 0 & 1.0631 & -0.4119 \\
0 & 0 & -0.4119 & 1.0631
\end{array}\right], \\
P_{x}=\left[\begin{array}{cccc}
0.2689 & -0.9436 & 0.2134 & -0.8727 \\
-0.9436 & 26.4751 & 0.0344 & 0.0597 \\
0.2134 & 0.0344 & 0.4366 & -0.5899 \\
-0.8727 & 0.0597 & -0.5899 & 5.9295
\end{array}\right] .
\end{gathered}
$$

Additionally, the LMI optimization gains of the integrated and separated FE-based FTC designs are listed in Table 2. As can be seen, the uncertainty attenuation level, which refers to the integrated approach, is much less than that of the separated approach. This latter loses a certain robustness degree against uncertainties illustrating the better multiplicative fault estimation and compensation using the integrated FE-based FTC approach.

We learned that the fault nonlinear function satisfies the Lipschitz condition. In this way, the admissible Lipschitz constant, which refers to the integrated approach, is greater than the one given by the separated approach, thus illustrating the superiority of the integrated FE-based FTC approach to treat a large range of fault nonlinear function.

It is worth pointing that simulation results are given with online multiplicative fault estimation and compensation for the closed-loop nonlinear system in the presence of uncertainties as $\xi(x, t)=0.1 \times 3 \sin (0.3 t)$ such that

(i) the sliding mode controller (14) is considered where $F_{a, 1}=F_{a, 2}=1, \quad N_{1}=N_{2}=\left[\begin{array}{lll}-0.7500 & 0.0463 & -0.7500\end{array}\right]$, and $\eta_{c}(t)=\hat{\rho}_{c}+0.1$ such that the adaptive term is given by $\dot{\hat{\rho}}_{S c}=2 \times\left\|S_{c}(t)\right\|$,

(ii) the initial conditions are $x_{10}=\Pi / 15, x_{20}=0.2$, $x_{30}=\Pi / 12, \quad x_{40}=0, \quad \widehat{\theta}_{1}(t=0)=\widehat{\theta}_{2}(t=0)=0, \quad$ and $\widehat{\rho}_{c}(t=0)=0$,

(iii) $\sigma_{1}=\sigma_{2}=10$ and $\Gamma_{1}=\Gamma_{2}=0.75 \times\left[\begin{array}{lll}1 & 1 & 1\end{array}\right]$.

From Figure 4, it is quite clear to see that the state estimation errors remain zero in finite time; that is, the T-S adaptive observer proposed in this paper can robustly estimate nonlinear system states with acceptable performances. Figure 5 illustrates the first multiplicative fault estimation error (partial loss of actuator effectiveness).

The results display that the proposed adaptive observer ((7), (8), and (9)), using the integrated FE-based FTC design, is capable of estimating multiplicative actuator fault with better performances compared to the separated FE-based FTC design. Note that abrupt changes of fault can generate small peak in Figure 5 at time $5 \mathrm{sec}$. Meanwhile, an abnormal 


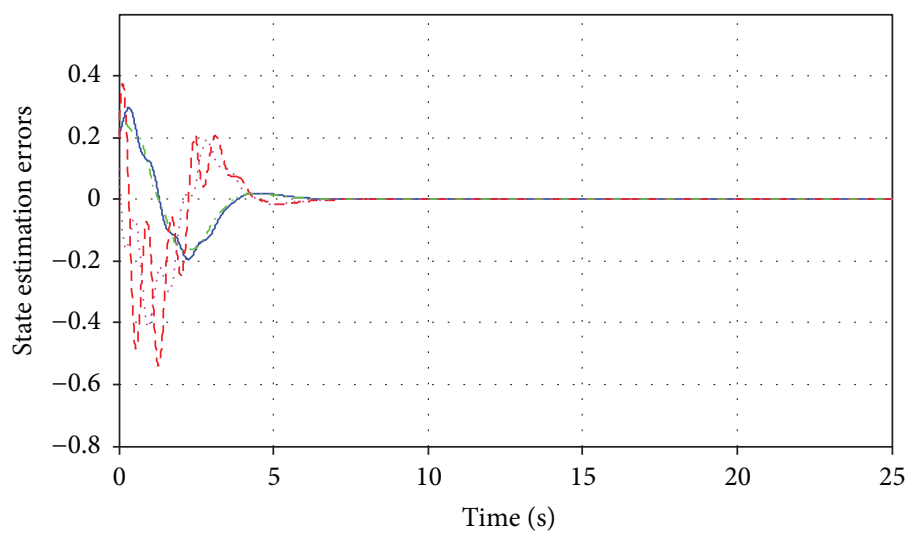

DC motor position velocity estimation error

Link position velocity DC motor angular velocity estimation error estimation error Link angular velocity estimation error

FIGURE 4: Nonlinear system state estimation errors.

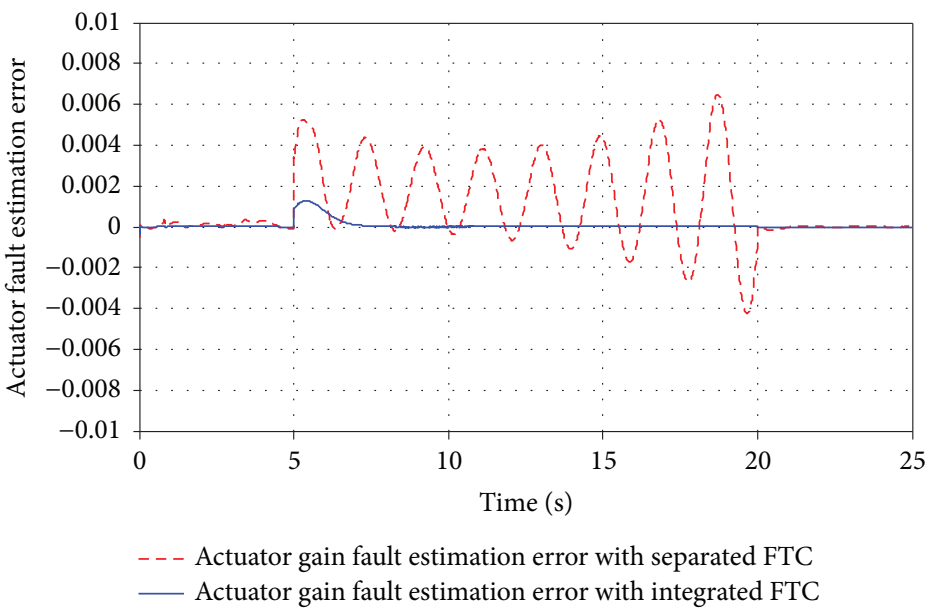

FIgURe 5: Actuator gain fault estimation error.

friction in the DC motor has been introduced, at $t=2.5 \mathrm{sec}$, to show the capability of the proposed FE strategy to handle process faults.

Consider Figure 6, which plots process fault estimation error generated using the separated FE-based FTC scheme against this variable using the integrated FE-based FTC scheme. It should be highlighted that the proposed adaptive observer design, using a single-step LMI formulation, can still track the considered process fault with better performances compared to the separated FE-based FTC design in terms of precision and robustness against the uncertainties.

Figures 7-9 outline a comparison between the nonlinear single-link flexible-joint robot output responses referring to two different cases: output responses with the separated FEbased FTC and output responses with the integrated FEbased FTC.

Based on the figures shown above, the conceived adaptive sliding mode controller (14) can stabilize with satisfactory performances the closed-loop nonlinear single-link flexiblejoint robot based on the integrated and separated FE-based sliding mode FTC. More precisely, as can be seen in zoomed versions from Figures 7-9, the proposed integrated FE-based FTC is capable of compensating real multiplicative faults with better performances compared to the separated FEbased FTC in terms of precision and robustness against uncertainties.

\section{Conclusion}

This paper has proposed two adaptive sliding mode FTC schemes for an uncertain nonlinear system subject to multiplicative and process faults. In the first scheme, the separated FE-based FTC is constructed to compensate real fault effects based on output feedback information and to ensure robust stability of the closed-loop system. In the second scheme, the integrated FE-based sliding mode FTC is conceived in 


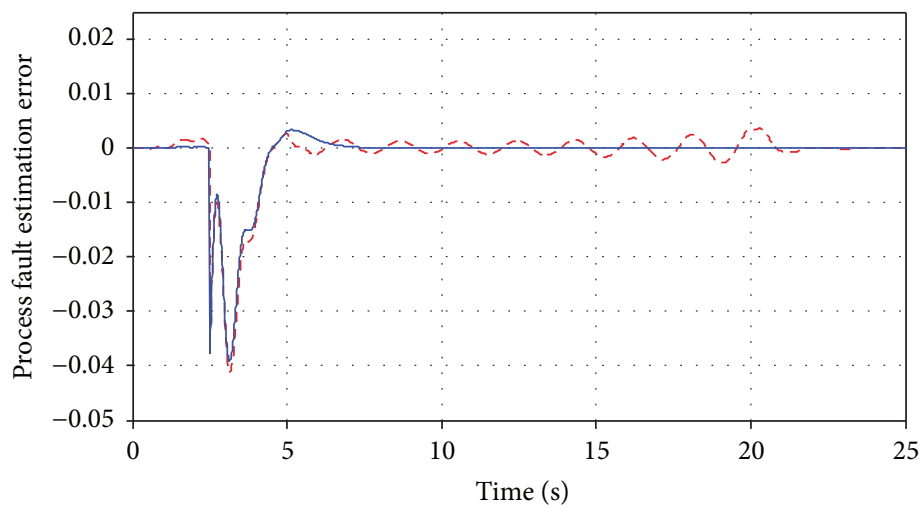

- - - Process fault estimation error with separated FTC

— Process faulte stimation error with integrated FTC

Figure 6: Process fault estimation error.

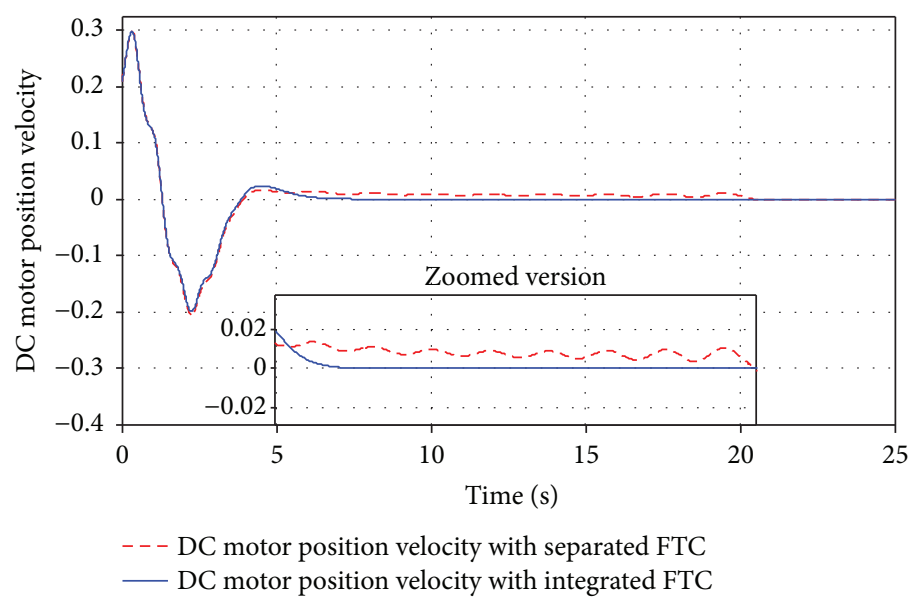

FIGURE 7: First closed-loop system output response: output response with the separated FE-based FTC (red line) and output response with the integrated FE-based FTC (blue line).

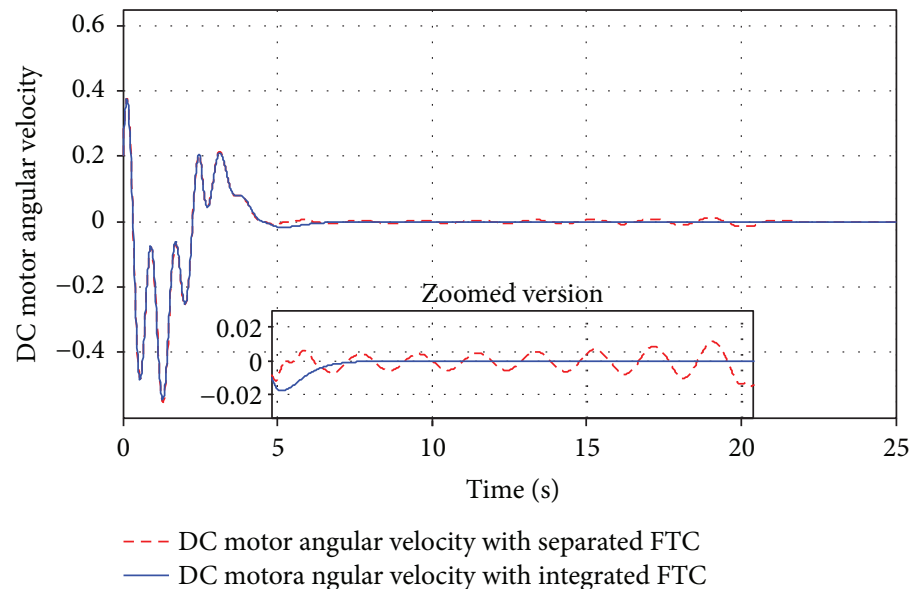

FIGURE 8: Second closed-loop system output response: output response with the separated FE-based FTC (red line) and output response with the integrated FE-based FTC (blue line). 


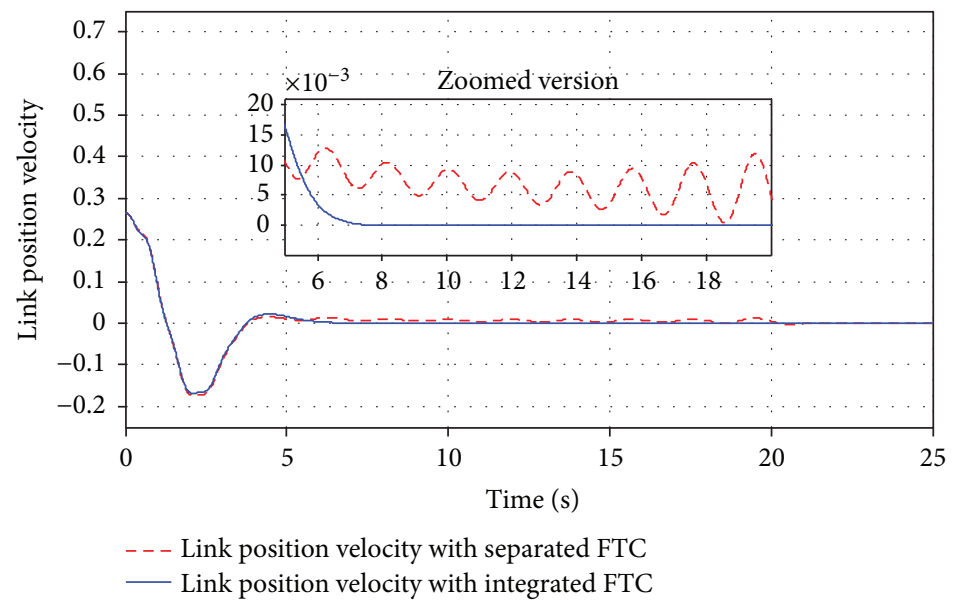

FIGURE 9: Third closed-loop system output response: output response with the separated FE-based FTC (red line) and output response with the integrated FE-based FTC (blue line).

order to achieve an optimal robustness interaction between observer and controller models. The novelty of the proposed approach is that the observer and controller gains are obtained by solving a single-step multiobjective LMI optimization problem in order to offer a solution to stabilize the closed-loop nonlinear system despite the occurrence of real fault effects. As shown in the comparative simulations of the flexible-joint robot arm system described by the T-S fuzzy model, the proposed integrated FE-based FTC improves the best robustness interactions between multiplicative fault estimation and sliding mode control. Summing up the results, we can conclude that the research into the integrated FEbased sliding mode FTC for an uncertain nonlinear system has been very successful.

\section{Data Availability}

The data used to support the findings of this study are available from the corresponding author upon request.

\section{Conflicts of Interest}

The authors declare that there is no conflict of interest regarding the publication of this paper.

\section{References}

[1] L. Wang, M. Cai, H. Zhang, F. Alsaadi, and L. Chen, "Active fault-tolerant control for wind turbine with simultaneous actuator and sensor faults," Complexity, vol. 2017, Article ID 6164841, 11 pages, 2017

[2] M. Van, P. Franciosa, and D. Ceglarek, "Fault diagnosis and fault-tolerant control of uncertain robot manipulators using high-order sliding mode," Mathematical Problems in Engineering, vol. 2016, Article ID 7926280, 14 pages, 2016.

[3] R. Isermann, Fault-Diagnosis Applications : Model-Based Condition Monitoring: Actuators, Drives, Machinery, Plants, Sensors, and Fault-Tolerant Systems, Springer, Science and Business Media, 2011.
[4] H. Alwi, C. Edwards, and C. P. Tan, Fault Detection and FaultTolerant Control Using Sliding Modes, Springer, Science and Business Media, 2011.

[5] J. Wang, " $\mathrm{H}_{\infty}$ fault-tolerant controller design for networked control systems with time-varying actuator faults," International Journal of Innovative Computing Information and Control, vol. 11, no. 4, pp. 1471-1481, 2015.

[6] F. Li, H. Zhenggao, and G. Zhao, "Fault estimation and adaptive fault tolerant control for dynamic systems based on the second-order sliding mode observer," Proceedings of the Institution of Mechanical Engineers, Part I: Journal of Systems and Control Engineering, vol. 230, no. 3, pp. 222-230, 2016.

[7] T. Takagi and M. Sugeno, "Fuzzy identification of systems and its applications to modeling and control," IEEE Transactions on Systems, Man, and Cybernetics, vol. SMC-15, no. 1, pp. 116-132, 1985.

[8] S. Aouaouda, M. Chadli, V. Cocquempot, and M. Tarek Khadir, "Multi-objective $\mathrm{H}_{-} / \mathrm{H}_{\infty}$ fault detection observer design for Takagi-Sugeno fuzzy systems with unmeasurable premise variables: descriptor approach," International Journal of Adaptive Control and Signal Processing, vol. 27, no. 12, pp. 10311047, 2013.

[9] H. Wang, D. Ye, and G. H. Yang, "Actuator fault diagnosis for uncertain T-S fuzzy systems with local nonlinear models," Nonlinear Dynamics, vol. 76, no. 4, pp. 1977-1988, 2014.

[10] D. Ichalal, B. Marx, J. Ragot, and D. Maquin, "Fault detection, isolation and estimation for Takagi-Sugeno nonlinear systems," Journal of the Franklin Institute, vol. 351, no. 7, pp. 3651-3676, 2014.

[11] G. H. Yang and H. Wang, "Fault detection and isolation for a class of uncertain state-feedback fuzzy control systems," IEEE Transactions on Fuzzy Systems, vol. 23, no. 1, pp. 139-151, 2015.

[12] A. B. Brahim, S. Dhahri, F. B. Hmida, and A. Sellami, "An $\mathrm{H}_{\infty}$ sliding mode observer for Takagi-Sugeno nonlinear systems with simultaneous actuator and sensor faults An," International Journal of Applied Mathematics and Computer Science, vol. 25, no. 3, pp. 547-559, 2015.

[13] A. Ben Brahim, S. Dhahri, F. Ben Hmida, and A. Sellami, "Simultaneous actuator and sensor faults reconstruction based on robust sliding mode observer for a class of nonlinear systems," Asian Journal of Control, vol. 19, no. 1, pp. 362-371, 2017. 
[14] R. Raoufi, H. J. Marquez, and A. S. I. Zinober, “ $\mathscr{H}_{\infty}$ sliding mode observers for uncertain nonlinear Lipschitz systems with fault estimation synthesis," International Journal of Robust and Nonlinear Control, vol. 20, pp. 1785-1801, 2010.

[15] S. Dhahri, A. Sellami, and F. B. Hmida, "Robust $H_{\infty}$ sliding mode observer design for fault estimation in a class of uncertain nonlinear systems with LMI optimization approach," International Journal of Control, Automation and Systems, vol. 10, no. 5, pp. 1032-1041, 2012.

[16] J. Zhang, S. K. Nguang, and A. K. Swain, "Detection and isolation of incipient sensor faults for a class of uncertain nonlinear systems," IET Control Theory and Applications, vol. 6, no. 12, pp. 1870-1880, 2012.

[17] A. Valibeygi, A. Toudeshki, and K. Vijayaraghavan, "Observer-based sensor fault estimation in nonlinear systems," Proceedings of the Institution of Mechanical Engineers, Part I: Journal of Systems and Control Engineering, vol. 230, no. 8, pp. 759-777, 2016.

[18] J. Lan and R. J. Patton, "Integrated fault estimation and faulttolerant control for uncertain Lipschitz nonlinear systems," International Journal of Robust and Nonlinear Control, vol. 27, no. 5, pp. 761-780, 2017.

[19] C. Lu, Y. Cheng, H. Liu, and Z. Wang, “An approach to fault detection and isolation for control components in the aircraft environmental control system," Proceedings of the Institution of Mechanical Engineers, Part G: Journal of Aerospace Engineering, vol. 228, no. 7, pp. 1202-1214, 2014.

[20] S. Yin, H. Luo, and S. X. Ding, "Real-time implementation of fault-tolerant control systems with performance optimization," IEEE Transactions on Industrial Electronics, vol. 61, no. 5, pp. 2402-2411, 2014.

[21] S. X. Ding, P. M. Frank, and E. L. Ding, "An approach to the detection of multiplicative faults in uncertain dynamic systems," in Proceedings of the 41st IEEE Conference on Decision and Control, 2002, pp. 4371-4376, Las Vegas, NV, USA, 2002.

[22] C. P. Tan and C. Edwards, "Multiplicative fault reconstruction using sliding mode observers," in 2004 5th Asian Control Conference (IEEE Cat. No.04EX904), pp. 957-962, Melbourne, VIC, Australia, 2004.

[23] K. Zhang, B. Jiang, and P. Shi, "Fast fault estimation and accommodation for dynamical systems," IET Control Theory \& Applications, vol. 3, no. 2, pp. 189-199, 2009.

[24] J. Lan and R. J. Patton, “A new strategy for integration of fault estimation within fault-tolerant control," Automatica, vol. 69, pp. 48-59, 2016.

[25] X. Zhang, M. M. Polycarpou, and T. Parisini, "Fault diagnosis of a class of nonlinear uncertain systems with Lipschitz nonlinearities using adaptive estimation," Automatica, vol. 46, no. 2, pp. 290-299, 2010.

[26] C. Gao and G. Duan, "Robust adaptive fault estimation for a class of nonlinear systems subject to multiplicative faults," Circuits, Systems, and Signal Processing, vol. 31, no. 6, pp. 2035-2046, 2012. 


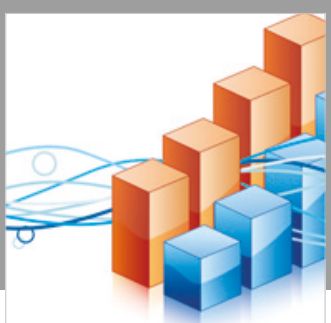

Advances in

Operations Research

\section{-n-m}
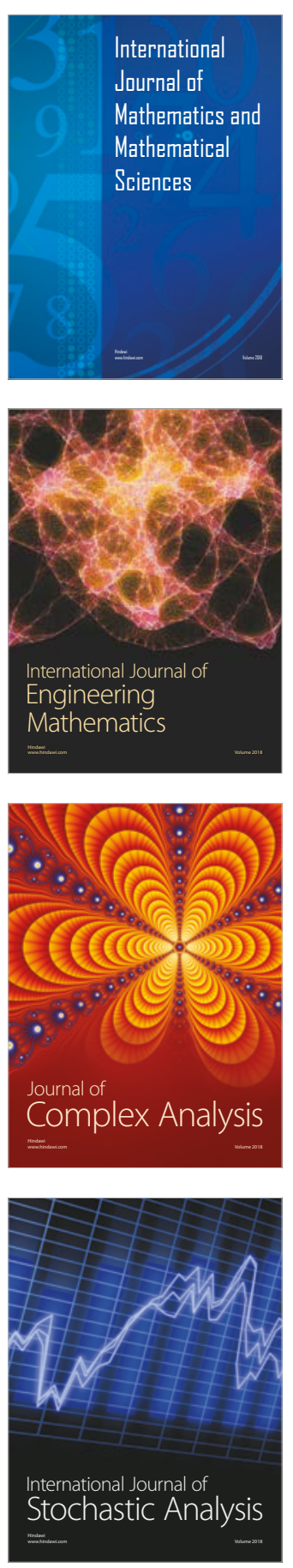
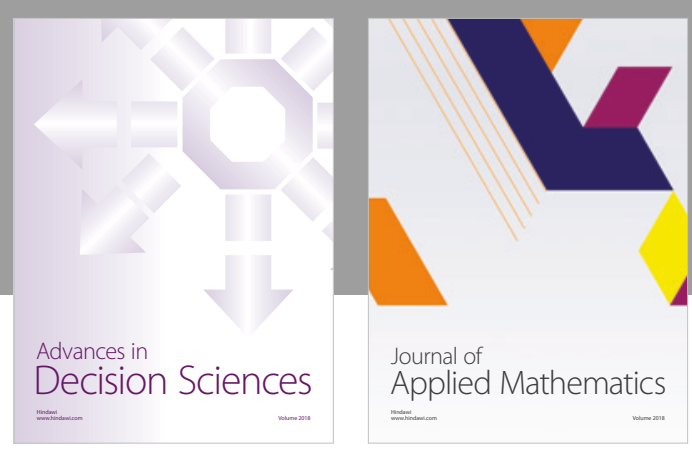

Journal of

Applied Mathematics
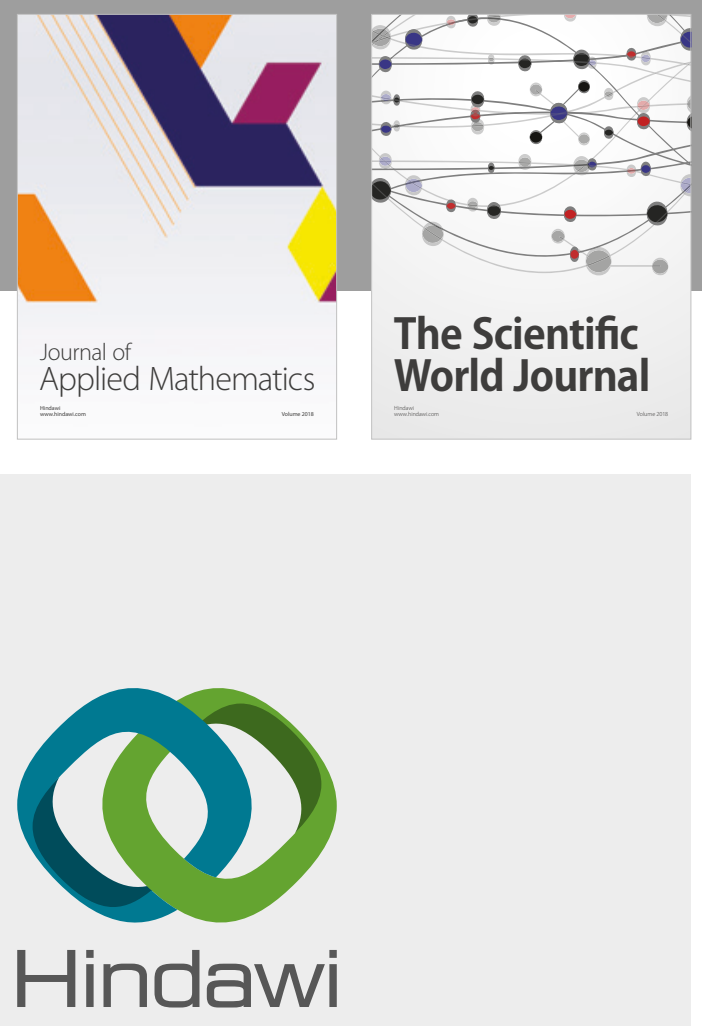

Submit your manuscripts at

www.hindawi.com

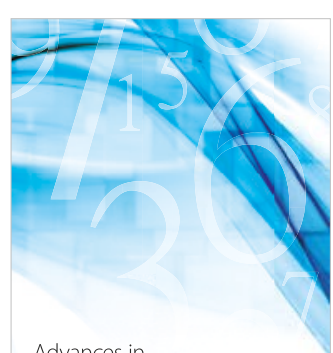

Advances in
Numerical Analysis
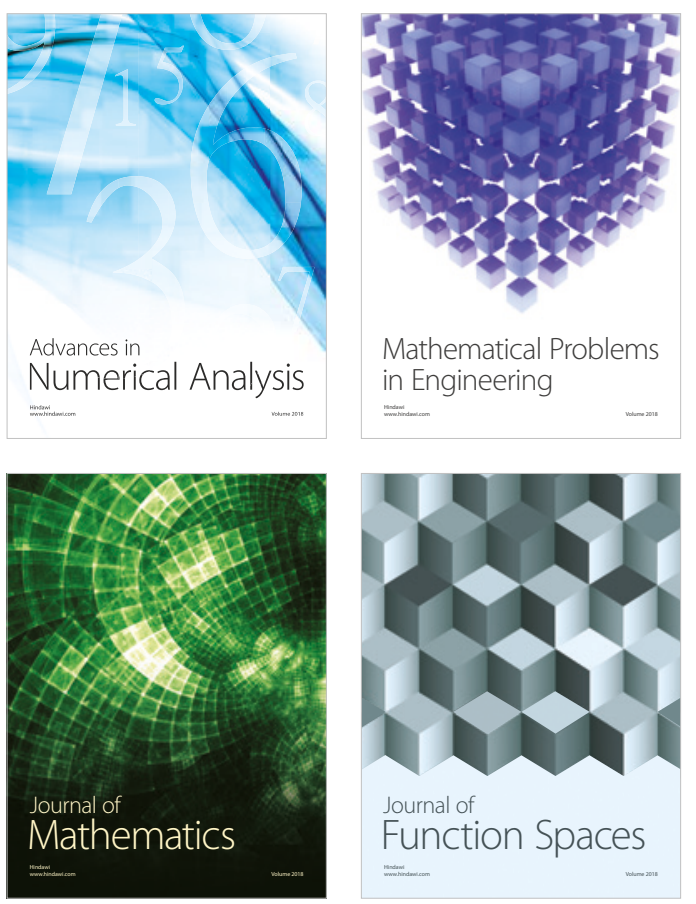

Mathematical Problems in Engineering

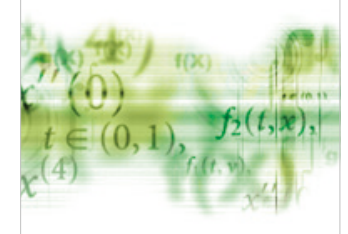

International Journal of

Differential Equations

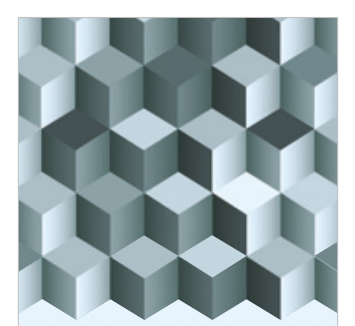

Journal of

Function Spaces
The Scientific

World Journal

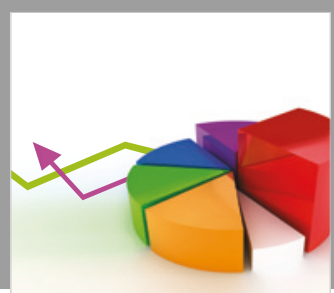

Journal of

Probability and Statistics
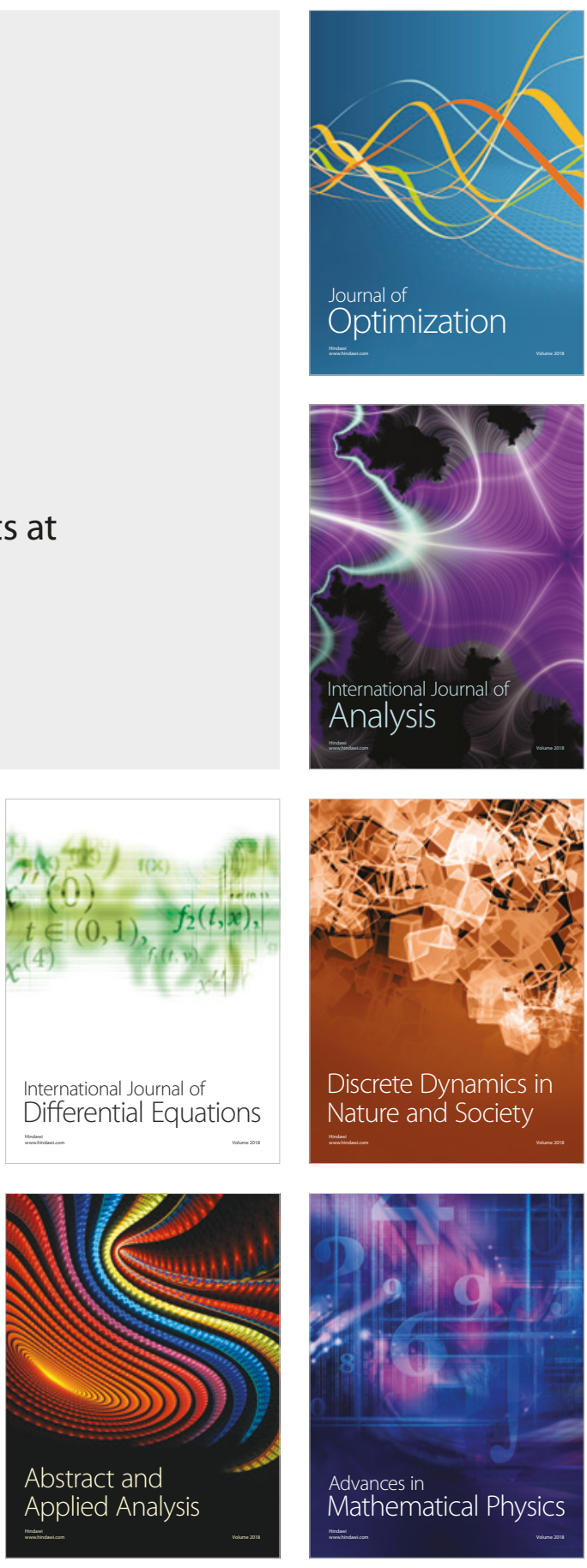